\section{Avaliação do acesso às ações de controle da tuberculose no contexto das equipes de saúde da família de Bayeux - PB*}

\section{Evaluation of access to tuberculosis control actions in the context of family health teams in Bayeux - PB}

\section{Alinne Beserra Lucena Marcolino' Jordana de Almeida Nogueira" Antonio Ruffino-Netto"' \\ Ronei Marcos Moraes ${ }^{\text {IV }}$ \\ Lenilde Duarte Sáv \\ Tereza Cristina Scatena Villa ${ }^{\text {VI }}$}

\section{Francieuldo Justino Rolim VII}

'Curso de Pós Graduação em Enfermagem e Saúde da Universidade Federal da Paraíba - UFPB.

"Departamento de Enfermagem Médico-cirúrgica, Programa de Pós-graduação em Enfermagem-Universidade Federal da Paraíba - UFPB.

I"'Departamento de Medicina Social da Faculdade de Medicina de Ribeirão Preto da USP. Coordenador da Rede Brasileira de Pesquisa em Tuberculose (REDE-TB)

IV Departamento de Estatística da Universidade Federal da Paraíba-UFPB.

${ }^{\vee}$ Departamento de Enfermagem em Saúde Pública e Psiquiátrica, Programa de Pós-graduação em Enfermagem da Universidade Federal da Paraíba - UFPB.

${ }^{v /}$ Coordenador da Área de Estudos Operacionais da Rede Brasileira de pesquisa em Tuberculose (REDE-TB) da Escola de Enfermagem de Ribeirão Preto da USP.

VII Graduado em Enfermagem pela Universidade Federal da Paraíba - UFPB.

*O artigo origina-se da dissertação de mestrado de Aline Beserra de Lucena Marcolino intitulada "Ações de Controle da Tuberculose no contexto das equipes de saúde da família de Bayeux-PB: avaliação dos componentes acesso e elenco de serviços", 2007-UFPB e se insere no projeto "Avaliação das dimensões organizacionais e de desempenho das equipes de saúde da família no controle da TB em dois municípios da região metropolitana da Paraíba", financiado pela FAPESQ/ MS/ $\mathrm{CNPq}-\mathrm{N}^{\circ}$ 078/07.

Correspondência: Alinne Beserra Lucena Marcolino. Rua Lindolfo José Correia das Neves $n^{\circ} 419$ apto 702 - Bessa, João Pessoa, CEP 58037-520. E-mail: alinneblucena@bol.com.br

\section{Resumo}

A descentralização das ações de controle da Tuberculose para o âmbito da Atenção Primária à Saúde (APS), vem impondo reorientação da prática das Equipes de Saúde da Família (ESF) e requerendo metodologias que avaliem em que medida os componentes da APS estão sendo alcançados. Este estudo toma como recorte um componente da APS-acesso, com o objetivo de avaliar as ações de controle da Tuberculose no contexto das ESF em Bayeux-PB. Pesquisa avaliativa, de abordagem quantitativa, que envolveu 82 profissionais de saúde. $\mathrm{O}$ instrumento utilizado continha sete perguntas fechadas, segundo possibilidades produzidas por escala intervalar tipo Likert. Os dados foram tabulados utilizando-se o programa - Statistical Package for the Social Sciences e analisados segundo frequência $\mathrm{e}$ mediana. Os resultados revelaram fragilidades e potencialidades de acesso às ações de controle da Tuberculose (TB). Quanto às potencialidades, constatou-se que a descentralização do tratamento dos casos de TB vem se estabelecendo na prática das ESF: $92,7 \%$ dos entrevistados mencionaram que os doentes de TB sempre conseguem consulta nas unidades, sendo possível para $82,9 \%$ obtê-la no prazo de 24 horas; os medicamentos específicos apresentaram-se acessíveis para $64,6 \%$ das ESF. Como fragilidades, verificou-se que $61 \%$ dos entrevistados não realizaram coleta de escarro; $54,9 \%$ das unidades não oferecem atendimento no horário de almoço; 89,8\% dos entrevistados não contam com auxílio transporte; apenas $40,2 \%$ das unidades adotam regularmente a visitação domiciliar. Recomenda-se a adoção de mecanismos de gestão que viabilizem a uniformização e utilização dos recursos existentes, ampliando a capacidade resolutiva das ESF, promovendo eficiência na prestação de serviços e assegurando o acesso da população.

Palavras-chave: Tuberculose. Atenção Primária à Saúde. Saúde da Família. 


\section{Abstract}

The decentralization of Tuberculosis control actions in Primary Health Care (PHC) has imposed the reorientation of practices of Family Health Teams (FHT) and required methods that evaluate to what extent the components of PHC are being reached. This study focuses on the PHC-access component, aimed at analyzing Tuberculosis control actions in the context of the FHT in Bayeux-PB. It is an evaluative study with a quantitative approach, involving 82 healthcare workers. The tool used had seven closed questions, according to the possibilities given by a Likert type interval scale. Data were tabulated using the software Statistical Package for the Social Sciences and were analyzed according to frequency and median. Outcomes revealed the weaknesses and potentialities of access to Tuberculosis control actions. Regarding potentialities, decentralization of treatment is being established in the practice of the FHT: $92.7 \%$ of the interviewees mentioned that Tuberculosis patients always obtain appointments at units, in that $82.9 \%$ may obtain it within 24 hours; specific medication was accessible to $64.6 \%$ of the FHT. As to weaknesses, $61 \%$ of interviewees did not collect sputum; $54.9 \%$ of units do not offer care during lunch time; $89.8 \%$ do not have support for transportation; only $40.2 \%$ adopt regular home visitation. Management mechanisms that enable to standardize and use existing resources to expand FHT resolution capability, promoting efficiency in the services, assuring population access is recommended.

Keywords: Tuberculosis. Primary Health Care. Family Health

\section{Introdução}

O Brasil vive, há mais de duas décadas, grandes mudanças na organização, financiamento e oferta de serviços de saúde, decorrentes principalmente da necessidade de se disponibilizar para a população serviços básicos, acessíveis, equitativos e de melhor qualidade ${ }^{1}$. Nesta perspectiva a institucionalização da Estratégia de Saúde da Família ganha evidência significativa, como instrumento de reorganização do sistema de serviços de saúde. A introdução desta estratégia como política de reordenação do setor saúde, exigiu reformulação de áreas programáticas até então estruturadas verticalmente. Programas cuja base histórica esteve alicerçada em diretrizes estabelecidas e coordenadas pela esfera federal e estadual, destacaram a necessidade de ampliar seus espaços de atuação utilizando como estratégia as equipes de saúde da família (ESF).

O Programa Nacional de Controle da Tuberculose introduziu novas possibilidades de intervenção em sua proposta de trabalho, assinalando que a inserção das ações de controle da tuberculose no âmbito das ESF poderia promover integração dos serviços, significando oportunidade para expansão das atividades de controle da doença ${ }^{2}$. Nesta direção, vem sendo proposto que os serviços de saúde se organizem para que as ESF incorporem em suas atividades a responsabilidade pelo desenvolvimento das ações de controle da tuberculose. Para tal devem estar capacitadas para realizar suspeição e diagnóstico dos casos; tratar e supervisionar a ingestão medicamentosa; acompanhar os contatos; manter atualizado o sistema de informação; realizar ações preventivas (BCG, quimioprofilaxia) e educativas junto à comunidade ${ }^{3}$.

Esta experiência vem se consolidando nas práticas de saúde de maneira gradativa, reconhecendo, entretanto, que os municípios agregam especificidades locais (políticas/organizacionais/humanas) que os tornam heterogêneos na dinâmica de enfrentamento da doença. No Estado da 
Paraíba, a descentralização das ações de controle da tuberculose (TB), vem se conformando à medida que se reorganiza a rede de assistência à saúde, articulada, sobretudo, com a ampliação da cobertura da Estratégia Saúde da Família ${ }^{4}$. Em 2005, foram avaliados os seis municípios considerados prioritários para o controle da TB na Paraíba (João Pessoa, Bayeux, Santa Rita, Campina Grande, Patos e Cajazeiras), evidenciando que, nos municípios menores e do interior, onde houve avanço na implantação e na expansão do número de ESF, as ações de controle da TB - em especial o Tratamento Supervisionado (TS) - foram incorporadas por estas equipes, repercutindo positivamente no aumento dos percentuais de cura da doença.

Nos municípios de pequeno porte, localizados no sertão e na região metropolitana, todas as equipes aderiram ao TS. Por outro lado, nos dois maiores municípios do Estado (João Pessoa e Campina Grande), apesar do aumento da cobertura das ESF, nem todas adotaram o TS. O estudo apontou ainda que a situação dos grandes centros urbanos do Estado da Paraíba deveria ser melhor investigada em termos das barreiras à incorporação das atividades de controle da TB pelas ESF ${ }^{4}$.

O município de Bayeux, pertencente à região metropolitana, apesar de apresentar características e problemas peculiares aos grandes centros urbanos, alcançou êxito na implantação das ações de controle da TB, registrando índices desejáveis de desfecho terapêutico. Em 2003, o percentual de cura alcançou 97,3\%, e taxa de abandono foi de $2,7 \%$. Em 2007, houve discreta redução na taxa de cura $(92,5 \%)$ e aumento da taxa de abandono (5\%), mas apesar disso índices superiores ao recomendado pelo Programa Nacional de Controle da Tuberculose, que preconiza curar pelo menos $85 \%$ dos casos. Neste mesmo período (2003-2007), o coeficiente de incidência de TB por todas as formas, diminuiu de 75,9 para 43,64 casos por 100.000 habitantes.

Os resultados satisfatórios alcançados pelo município de Bayeux são atribuídos principalmente à atuação das equipes de saúde da família $(\mathrm{ESF})^{4}$. Destaca-se, entretanto, que, apesar dos avanços, o município de Bayeux apresentou em 2007 coeficiente de incidência superior ao registrado no Estado da Paraíba ( 32,4 por 100.000 habitantes).

Nesta direção reconhece-se que a descentralização das ações de saúde para o âmbito das ESF vem requerendo a adoção de pesquisas e metodologias que permitam avaliar o alcance desta estratégia e o impacto destas inovações na organização dos serviços da atenção primária à saúde $(\mathrm{APS})^{5}$. A "avaliação da incorporação de novas práticas sanitárias na rotina de profissionais" possibilita monitorar a capacidade dos serviços em responder às necessidades de saúde, acompanhar os efeitos das intervenções, identificar e corrigir problemas e retroalimentar equipes de saúde, gestores, políticos e comunidade ${ }^{6}$.

Portanto, nesta perspectiva, a avaliação não compreende apenas a identificação de problemas ou o diagnóstico de uma determinada situação, mas, com base no que foi avaliado, mudar a realidade, procurando seu aperfeiçoamento. Caracteriza-se como um poderoso instrumento de mudança, sendo seu maior objetivo identificar, analisar e corrigir situações potencialmente passíveis de melhoria?

Diante dos aspectos citados e compreendendo a relevância da avaliação como uma ferramenta de aprimoramento do Sistema Único de Saúde, enfatiza-se a necessidade de avaliar em que medida os componentes da APS (primeiro contato, integralidade, longitudinalidade e coordenação) vêm sendo alcançados.

Este estudo está direcionado para a avaliação do cumprimento de um componente da APS - o acesso - no tocante às ações de controle da TB desenvolvidas no contexto das ESF de Bayeux- PB.

\section{Metodologia}

$\mathrm{O}$ atual projeto insere-se na pesquisa intitulada “Avaliação das dimensões organi- 
zacionais e de desempenho das equipes de saúde da família no controle da tuberculose em dois municípios da região metropolitana da Paraíba", aprovada pela FAPESQ, TC 078/07. Trata-se de pesquisa avaliativa, de abordagem quantitativa, que utilizou o desenho de estudo seccional, tendo como quadro teórico as dimensões da Atenção Primária em Saúde.

Elegeu-se como local de estudo o município de Bayeux, pertencente à região metropolitana da Paraíba. Segundo estimativa do IBGE (2004), a população residente no município é de 91.646 habitantes, com elevado grau de urbanização $(99,6 \%)^{8}$. No âmbito da atenção básica, o município conta atualmente com 28 Equipes de Saúde da Família, distribuídas em cinco Distritos Sanitários de Saúde, sendo responsáveis pela cobertura de $92 \%$ da população.

Ressalta-se que está previsto que cada ESF siga composta por 1(um) profissional médico, 1 (um) enfermeiro e 1 (um) auxiliar de enfermagem. A quantidade de agentes comunitários de saúde é variável. Portanto, a intenção voltou-se a principio para a realização de estudo populacional, em que fossem incluídos todos os profissionais médicos ( $n=28)$, todos os enfermeiros $(n=28) e$ todos os auxiliares de enfermagem $(n=28)$. Quanto aos agentes comunitários de saúde (ACS), a seleção foi precedida de alguns critérios, tais como: tempo de atuação (mais de dois anos como ACS), ter experiência e treinamento na área de TB. Considerando que as equipes locais direcionam as funções de cada ACS, vinculando-os às diferentes áreas programáticas da atenção básica, a participação do ACS foi definida segundo indicação do profissional enfermeiro, na perspectiva de que o mesmo pudesse ter subsídios para contribuir com a pesquisa. Deste modo foi indicado um ACS por equipe de saúde ( $\mathrm{n}=28)$.

Logo, a intenção seria investigar 112 profissionais. Entretanto, por ocasião da coleta de dados nem todas as equipes estavam completas; alguns profissionais encontravam-se afastados de suas funções (licença médica, gestante) e outros se recusaram a participar da pesquisa, alegando pouca proximidade com a temática, falta de experiência em TB ou falta de tempo. Assim, a população investigada constituiu-se de 84 profissionais, conforme demonstrado no Quadro 1.

É importante informar que, em uma das equipes pertencente à área do Distrito III, foram entrevistados apenas dois profissionais, cujos questionários apresentaram disparidade nas respostas. $\mathrm{Na}$ escala de possibilidades de respostas, um dos entrevistados registrou que "sempre" determinada ação era realizada; em contrapartida, o outro profissional respondeu que esta mesma ação "nunca" era realizada. Não havendo um terceiro entrevistado para confrontar as informações registradas, optou-se por descartá-los do estudo, para evitar inconsistência na análise. Justifica-se, portanto, a redução de "n", culminando em um total de 82 profissionais. Cabe destacar que as perdas ocorridas não invalidam os resultados obtidos.

Quadro 1 - Distribuição dos profissionais que constituíram a amostra segundo Distritos de Saúde do Município de Bayeux, PB

Chart 1 - Distribution of the professionals included in the sample per Health District in the City of Bayeux, $P B$

\begin{tabular}{|lcccccc|}
\hline Profissional & Distrito I & Distrito II & Distrito III & Distrito IV & Distrito V & Total \\
\hline Médico & 3 & 4 & 3 & 4 & 3 & 17 \\
Enfermeiro & 5 & 7 & 3 & 5 & 2 & 22 \\
ACS & 5 & 6 & 3 & 5 & 3 & 22 \\
Auxiliar & 5 & 7 & 4 & 5 & 2 & 23 \\
\hline Total & 18 & 24 & 13 & 19 & 10 & 84 \\
\hline
\end{tabular}


Para a coleta dos dados foi utilizado um questionário estruturado, elaborado por Makinko e Almeida ${ }^{9}$ e adaptado por Villa e Ruffino $^{10}$, contendo indicadores de avaliação das ações de controle da TB segundo componentes da APS. O questionário foi dividido em seções, cada uma contemplando questões relacionadas a um determinado tema, sendo que uma delas se referiu ao componente "acesso às ações de controle da TB" utilizado no presente estudo. Este instrumento foi adaptado em equipe mediante reuniões contínuas, testado em uma amostra de profissionais e readequado posteriormente ${ }^{10}$.

Para contemplar o componente "acesso" foram utilizados sete indicadores específicos. Os entrevistados responderam cada pergunta segundo uma escala de possibilidades preestabelecida, escala de Likert, à qual foi atribuído um valor de acordo com as possibilidades de respostas: 1 (nunca); 2 (quase nunca); 3 (algumas vezes); 4 (muitas vezes); 5 (quase sempre); 6 (sempre) e 88 (não respondeu).

A coleta de dados ocorreu nos meses de abril e maio de 2007. No primeiro momento foram apresentados os objetivos da pesquisa, e após o consentimento foi assinado o Termo de Consentimento Livre e Esclarecido. Ressalta-se que era intenção da pesquisadora aplicar o questionário de forma que a mesma pudesse interagir com o entrevistado, explicitando as questões, esclarecendo as dúvidas que porventura ocorressem no momento do preenchimento do instrumento. Visto que a coleta de dados foi realizada no local de trabalho dos profissionais, identificaram-se obstáculos que poderiam interferir na rotina de serviço das equipes. Logo, objetivando minimizar a desistência dos entrevistados, optou-se pelo método autoaplicado.

A pesquisa foi apreciada pelo Comitê de Ética em Pesquisa do Centro de Ciências da Saúde da Universidade Federal da Paraíba, obtendo aprovação em 28/02/2007 sob o protocolo de $\mathrm{n}^{\circ}$. 886/07.

Os dados foram tabulados utilizando-se o software Statistical Package for the Social
Sciences (SPSS, versão 11.5 for Windows), e analisados estatisticamente, contemplando valores absolutos e relativos a cada Distrito de Saúde investigado. Posteriormente, foram analisados com base em uma medida de tendência central: a mediana.

É necessário informar que a mediana segue um escore de valores, representando, portanto, o menor escore (escore 1) o pior indicador, posto que concebe a idéia de que o acesso "nunca" ocorre. Da mesma forma, o escore de maior valor (escore 6) representa um indicador positivo, uma vez que concebe a ideia de que "sempre" tal indicador se encontra acessível ao usuário.

\section{Resultados}

A Tabela 1 apresenta a disponibilidade das Unidades pesquisadas quanto à realização da coleta de escarro para diagnóstico e tratamento da tuberculose. Foi constatado que $61 \%$ dos entrevistados mencionaram "nunca" realizar o procedimento na unidade de saúde da família. Apenas 12,2 \% dos entrevistados assinalaram "sempre" adotar tal procedimento. Ao analisar por distrito de saúde, verificou-se que no Distrito II este percentual atingiu $83,3 \%$. No Distrito III, observou-se que as opiniões divergiam, quando 54,5\% mencionaram que "nunca" realizaram o procedimento e $45,5 \%$ representam a somatória das opções "muitas vezes", "quase sempre" e "sempre".

Outro aspecto pesquisado refere-se ao horário de trabalho das equipes e de funcionamento das unidades de saúde. Em geral, o horário de atendimento se restringe ao período de $7 \mathrm{~h}$ às $11 \mathrm{~h}$ e das $13 \mathrm{~h}$ às $17 \mathrm{~h}$, ficando a unidade fechada no horário de almoço (11h às 13h) ( Tabela 2).

Do total de entrevistados, $54,9 \%$ referiram que, neste horário, "nunca ocorre atendimento". No Distrito II, este percentual atingiu $75 \%$. No Distrito I, as respostas mostraram-se divergentes, sendo que $44,4 \%$ informaram "nunca" ocorrer atendimento no horário de almoço, e 27,8\% informaram que "sempre" o paciente é atendido neste horário. Neste mesmo distrito, 16,7\% não 
Tabela 1 - Distribuição percentual referente à realização na unidade do exame de escarro em indivíduos sintomáticos e doentes nos distritos de saúde de Bayeux, segundo os profissionais da ESF. Paraíba, 2007.

Table 1 - Percentage distribution of sputum smear examinations in symptomatic individuals/patients in the health districts of Bayeux, according to Family Health Team practitioners. Paraiba, 2007

\begin{tabular}{|c|c|c|c|c|c|c|c|c|c|c|c|c|c|c|}
\hline \multirow{2}{*}{ Distrito } & \multicolumn{2}{|c|}{ Nunca } & \multicolumn{2}{|c|}{ Quase nunca } & \multicolumn{2}{|c|}{ Algumas vezes } & \multicolumn{2}{|c|}{ Muitas vezes } & \multicolumn{2}{|c|}{ Quase sempre } & \multicolumn{2}{|c|}{ Sempre } & \multicolumn{2}{|c|}{ Não Respondeu } \\
\hline & $\mathrm{n}$ & $\%$ & $\mathrm{n}$ & $\%$ & $\mathrm{n}$ & $\%$ & $\mathrm{n}$ & $\%$ & $\mathrm{~N}$ & $\%$ & $\mathrm{n}$ & $\%$ & $\mathrm{n}$ & $\%$ \\
\hline Distrito I & 12 & 66,7 & & & & & 3 & 16,7 & & & 1 & 5,6 & 2 & 11,1 \\
\hline Distrito II & 20 & 83,3 & 1 & 4,2 & & & & & & & 3 & 12,5 & & \\
\hline Distrito III & 6 & 54,5 & & & & & 1 & 9,1 & 2 & 18,2 & 2 & 18,2 & & \\
\hline Distrito IV & 8 & 42,1 & 3 & 15,8 & 2 & 10,5 & 3 & 15,8 & & & 3 & 15,8 & & \\
\hline Distrito V & 4 & 40,0 & 1 & 10,0 & 3 & 30,0 & & & & & 1 & 10,0 & 1 & 10,0 \\
\hline Total & 50 & 61,0 & 5 & 6,1 & 5 & 6,1 & 7 & 8,5 & 2 & 2,4 & 10 & 12,2 & 3 & 3,7 \\
\hline
\end{tabular}

Fonte/Source: ESF Bayeux - PB/ Abril-Maio 2007.

Tabela 2 - Distribuição percentual referente à oferta de atendimento ao doente no horário de almoço, segundo os profissionais das ESF por Distrito de Saúde. Bayeux, PB.

Table 2 - Percentage distribution of patient care availability during lunch time, according to Family Health Team, per Health District. Bayeux, PB

\begin{tabular}{|c|c|c|c|c|c|c|c|c|c|c|c|c|c|c|}
\hline \multirow{2}{*}{ Distrito } & \multicolumn{2}{|c|}{ Nunca } & \multicolumn{2}{|c|}{ Quase nunca } & \multicolumn{2}{|c|}{ Algumas vezes } & \multicolumn{2}{|c|}{ Muitas vezes } & \multicolumn{2}{|c|}{ Quase sempre } & \multicolumn{2}{|c|}{ Sempre } & \multicolumn{2}{|c|}{ Não Respondeu } \\
\hline & $\mathrm{n}$ & $\%$ & $\mathrm{n}$ & $\%$ & $\mathrm{n}$ & $\%$ & $\mathrm{n}$ & $\%$ & $\mathrm{n}$ & $\%$ & $\mathrm{n}$ & $\%$ & $\mathrm{n}$ & $\%$ \\
\hline Distrito I & 8 & 44,4 & & & & & 1 & 5,6 & 1 & 5,6 & 5 & 27,8 & 3 & 16,7 \\
\hline Distrito II & 18 & 75,0 & & & & & & & 1 & 4,2 & 3 & 12,5 & 2 & 8,3 \\
\hline Distrito III & 5 & 45,5 & 3 & 27,3 & 1 & 9,1 & 2 & 18,2 & & & & & & \\
\hline Distrito IV & 9 & 47,4 & 2 & 10,5 & 2 & 10,5 & & & 4 & 21,1 & & & 2 & 10,5 \\
\hline Distrito V & 5 & 50,0 & 1 & 10,0 & 2 & 20,0 & & & 2 & 20,0 & & & & \\
\hline Total & 45 & 54,9 & 6 & 7,3 & 5 & 6,1 & 3 & 3,7 & 8 & 9,8 & 8 & 9,8 & 7 & 8,5 \\
\hline
\end{tabular}

Fonte/Source: ESF Bayeux - PB/ Abril-Maio 2007.

responderam a esta questão. Tal disparidade de resposta entre as ESF sugere que possivelmente, em algumas USF, se estabelece uma escala de trabalho que permite o rodízio dos profissionais e o funcionamento neste horário.

Quanto à disponibilidade para consulta médica aos pacientes de tuberculose, a Tabela 3 demonstra que $92,7 \%$ dos entrevistados informaram que os doentes de TB sempre podem conseguir uma consulta nas respectivas USF. O Distrito I atingiu maior percentual, assinalando que, em $100 \%$ dos casos, os doentes de TB conseguem uma consulta na unidade. Por outro lado, não foram assinaladas as respostas "nunca" e "quase nunca".

Quanto à agilidade em se obter uma consulta médica para os doentes de TB, $82,9 \%$ dos pesquisados referiram ser possível no prazo de 24 horas (Tabela 4). Nos Distritos I, II, III e IV, foi mencionado que "quase sempre" ocorre esta possibilidade. Ao somar as respostas "sempre" e "quase sempre”, obtém-se um percentual de $92,7 \%$, caracterizando resultado positivo de acesso ao atendimento. No Distrito IV, o percentual de resposta "sempre" alcançou 94,7\% dos entrevistados. Este é um importante indicador de acesso, principalmente, aos pacientes que se encontram em tratamento de TB.

É comum o aparecimento de reações adversas aos medicamentos na fase terapêutica inicial. Este fato implica em cuidados e atenção especial da equipe de saúde, uma vez que pode comprometer a adesão, 
Tabela 3 - Distribuição percentual referente à oferta de consulta aos doentes de TB segundo profissionais das ESF, por Distrito de Saúde. Bayeux, PB

Table 3 - Percentage distribution of availability of medical appointments for TB patients according to Family Health Teams, per Health District. Bayeux, PB.

\begin{tabular}{lcccccc}
\hline \multirow{2}{*}{ Distrito } & \multicolumn{2}{c}{ Algumas vezes } & \multicolumn{2}{c}{ Quase sempre } & \multicolumn{2}{c}{ Sempre } \\
\cline { 2 - 7 } & $\mathrm{n}$ & $\%$ & $\mathrm{n}$ & $\%$ & $\mathrm{n}$ & $\%$ \\
\hline Distrito I & & & & 18 & 100,0 \\
Distrito II & & 2 & 8,3 & 22 & 91,7 \\
Distrito III & & 2 & 18,2 & 9 & 81,8 \\
Distrito IV & 1 & 5,3 & & & 18 & 94,7 \\
Distrito V & & & 1 & 10,0 & 9 & 90,0 \\
\hline Total & 1 & 1,2 & 5 & 6,1 & 76 & 92,7 \\
\hline
\end{tabular}

Fonte/Source: ESF Bayeux - PB/ Abril-Maio 2007.

ocasionando o abandono do tratamento. Apenas 2 (dois) entrevistados (Distritos III e V) mencionaram que "nunca" os doentes de TB conseguem consulta médica no prazo de 24 horas.

Apesar da agilidade informada em se obter uma consulta médica, apenas $64,6 \%$ dos entrevistados mencionaram obter suprimento e oferta adequada de medicamentos para o tratamento da TB (Tabela 5). No Distrito I, 88,9\% assinalaram que "sempre" dispõem de suprimento medicamentoso para tratamento da TB. No Distrito III, observou-se maior divergência na opinião dos entrevistados, já que todas as possibilidades de respostas foram assinaladas. Neste mesmo Distrito, 27,3\% dos entrevistados registraram que "quase nunca" contam com suprimento de medicamentos para TB, e 27,3\% não responderam à pergunta. É relevante apontar que, neste indicador de acesso, 9,8\% dos entrevistados deixaram de opinar.

Um outro aspecto reconhecido como facilitador à adesão terapêutica está relacionado à viabilização de recursos que incentivem o comparecimento do paciente ao tratamento medicamentoso supervisionado na USF. A Tabela 6 demonstra que 89,0\% dos entrevistados "nunca" dispõem de vale-transporte para a realização do TS. Nos Distritos III e IV, $100 \%$ dos entrevistados assinalaram que as unidades não oferecem vale-transporte para o doente fazer o TS. Entretanto, nos Distritos I e II, uma parcela dos entrevistados não opinou sobre a

Tabela 4 - Distribuição percentual referente à obtenção de consulta médica no prazo de 24hs, caso o paciente sinta-se mal, segundo os profissionais das ESF do município de Bayeux, PB.

Table 4 - Percentage distribution of success in scheduling a medical appointment within 24 hours, when the patient feels ill, according to Family Health Teams. Bayeux, PB.

\begin{tabular}{|c|c|c|c|c|c|c|c|c|c|c|}
\hline \multirow{2}{*}{ Distrito } & \multicolumn{2}{|c|}{ Nunca } & \multicolumn{2}{|c|}{ Muitas vezes } & \multicolumn{2}{|c|}{ Quase sempre } & \multicolumn{2}{|c|}{ Sempre } & \multicolumn{2}{|c|}{ Não Respondeu } \\
\hline & $\mathrm{n}$ & $\%$ & $\mathrm{n}$ & $\%$ & $\mathrm{n}$ & $\%$ & $\mathrm{n}$ & $\%$ & $\mathrm{n}$ & $\%$ \\
\hline Distrito I & & & & & 2 & 11,1 & 16 & 88,9 & & \\
\hline Distrito II & & & 1 & 4,2 & 4 & 16,7 & 18 & 75,0 & 1 & 4,2 \\
\hline Distrito III & 1 & 9,1 & 1 & 9,1 & 1 & 9,1 & 8 & 72,7 & & \\
\hline Distrito IV & & & & & 1 & 5,3 & 18 & 94,7 & & \\
\hline Distrito V & 1 & 10,0 & & & & & 8 & 80,0 & 1 & 10,0 \\
\hline Total & 2 & 2,4 & 2 & 2,4 & 8 & 9,8 & 68 & 82,9 & 2 & 2,4 \\
\hline
\end{tabular}

Fonte/Source: ESF Bayeux - PB/ Abril-Maio 2007. 
Tabela 5 - Distribuição percentual das respostas dos entrevistados referente a suprimento e oferta adequada de medicamentos para o tratamento da TB, segundo Distrito de Saúde, no município de Bayeux, PB.

Table 5 - Percentage distribution of answers of interviewees about the adequate supply and offer of medications for TB, per Health District. Bayeux, PB

\begin{tabular}{|c|c|c|c|c|c|c|c|c|c|c|c|c|c|c|}
\hline \multirow{2}{*}{ Distrito } & \multicolumn{2}{|c|}{ Nunca } & \multicolumn{2}{|c|}{ Quase nunca } & \multicolumn{2}{|c|}{ Algumas vezes } & \multicolumn{2}{|c|}{ Muitas vezes } & \multicolumn{2}{|c|}{ Quase sempre } & \multicolumn{2}{|c|}{ Sempre } & \multicolumn{2}{|c|}{ Não Respondeu } \\
\hline & $\mathrm{n}$ & $\%$ & $n$ & $\%$ & $n$ & $\%$ & $\mathrm{n}$ & $\%$ & $\mathrm{n}$ & $\%$ & $\mathrm{n}$ & $\%$ & $\mathrm{n}$ & $\%$ \\
\hline Distrito I & & & & & & & & & 2 & 11,1 & 16 & 88,9 & & \\
\hline Distrito II & & & & & 5 & 20,8 & & & 3 & 12,5 & 15 & 62,5 & 1 & 4,2 \\
\hline Distrito III & 1 & 9,1 & 3 & 27,3 & 1 & 9,1 & 1 & 9,1 & 1 & 9,1 & 1 & 9,1 & 3 & 27,3 \\
\hline Distrito IV & & & & & & & & & 1 & 5,3 & 15 & 78,9 & 3 & 15,8 \\
\hline Distrito V & & & & & 1 & 10,0 & 1 & 10,0 & 1 & 10,0 & 6 & 60,0 & 1 & 10,0 \\
\hline Total & 1 & 1,2 & 3 & 3,7 & 7 & 8,5 & 2 & 2,4 & 8 & 9,8 & 53 & 64,6 & 8 & 9,8 \\
\hline
\end{tabular}

Fonte/Source: ESF Bayeux - PB/ Abril-Maio 2007.

Tabela 6 - Distribuição percentual referente à oferta de vale-transporte para o doente fazer o TS, segundo ESF dos Distritos de Saúde de Bayeux, PB.

Table 6 - Percentage distribution of free transportation tickets for patients to come for ST, according to Family Health Teams, per Health District. Bayeux, PB.

\begin{tabular}{lcccccc}
\hline Distrito & \multicolumn{2}{c}{ Nunca } & \multicolumn{2}{c}{ Quase nunca } & \multicolumn{2}{c}{ Não Respondeu } \\
\cline { 2 - 7 } & $\mathrm{n}$ & $\%$ & $\mathrm{n}$ & $\%$ & $\mathrm{~N}$ & $\%$ \\
\hline Distrito I & 14 & 77,8 & & & 4 & 22,2 \\
Distrito II & 21 & 87,5 & & & 3 & 12,5 \\
Distrito III & 11 & 100,0 & & & & \\
Distrito IV & 19 & 100,0 & & & & \\
Distrito V & 8 & 80,0 & 2 & 20,0 & & \\
\hline Total & 73 & 89,0 & 2 & 2,4 & 7 & 8,5 \\
\hline
\end{tabular}

Fonte/Source: ESF Bayeux - PB/ Abril-Maio 2007.

questão (22,2\% e $12,5 \%$ respectivamente). Neste caso, o recurso seria condição indispensável, uma vez que está previsto que a supervisão terapêutica ocorra diariamente, ou três vezes por semana.

Todavia, a inexistência de auxíliotransporte não inviabiliza a realização do tratamento supervisionado (TS), caso a equipe de saúde se disponha a realizar a supervisão terapêutica no domicílio.

A Tabela 7 demonstra que esta prática parece ser usual para uma parcela dos profissionais pesquisados.

Do total dos entrevistados, $40 \%$ registraram que os profissionais "sempre" vão ao domicílio do paciente, $6,1 \%$ "quase sempre", $12,2 \%$ "muitas vezes" e $34,1 \%$ "algumas vezes"; apenas, $4,9 \%$ referiram que eles "nunca" comparecem ao domicílio do paciente. Nos Distritos I e III, as respostas se polarizaram entre "algumas vezes" e "sempre”. No Distrito IV, as opiniões divergiram, sendo assinaladas todas as possibilidades de respostas. O Distrito II apresentou melhor desempenho, alcançando $50 \%$ dos entrevistados.

Cabe ressaltar que este estudo não analisou os resultados diferenciando as opiniões por categoria profissional. Considerando que $25 \%$ da amostra constituíram-se de ACS, estes resultados podem indicar que as respostas "sempre" tenham sido registradas predominantemente por esta categoria profissional, uma vez que a visita domiciliar é uma atribuição inerente à função do mesmo. 
Tabela 7 - Distribuição percentual referente à frequência com que os profissionais das ESF comparecem ao domicílio do doente. Bayeux, PB

Table 7 - Percentage distribution of the frequency with which Family Health Team professionals go to patients' homes.

Bayeux, $P B$

\begin{tabular}{|c|c|c|c|c|c|c|c|c|c|c|c|c|c|c|}
\hline \multirow{2}{*}{ Distrito } & \multicolumn{2}{|c|}{ Nunca } & \multicolumn{2}{|c|}{ Quase nunca } & \multicolumn{2}{|c|}{ Algumas vezes } & \multicolumn{2}{|c|}{ Muitas vezes } & \multicolumn{2}{|c|}{ Quase sempre } & \multicolumn{2}{|c|}{ Sempre } & \multicolumn{2}{|c|}{ Não Respondeu } \\
\hline & $\mathrm{n}$ & $\%$ & $\mathrm{n}$ & $\%$ & $\mathrm{n}$ & $\%$ & $n$ & $\%$ & $n$ & $\%$ & $n$ & $\%$ & $\mathrm{n}$ & $\%$ \\
\hline Distrito I & 1 & 5,6 & & & 9 & 50,0 & & & & & 8 & 44,4 & & \\
\hline Distrito II & & & & & 5 & 20,8 & 4 & 16,7 & 2 & 8,3 & 12 & 50,0 & 1 & 4,2 \\
\hline Distrito III & & & & & 5 & 45,5 & & & 1 & 9,1 & 5 & 45,5 & & \\
\hline Distrito IV & 2 & 10,5 & 1 & 5,3 & 7 & 36,8 & 3 & 15,8 & 1 & 5,3 & 5 & 26,3 & & \\
\hline Distrito V & 1 & 10,0 & & & 2 & 20,0 & 3 & 30,0 & 1 & 10,0 & 3 & 30,0 & & \\
\hline Total & 4 & 4,9 & 1 & 1,2 & 28 & 34,1 & 10 & 12,2 & 5 & 6,1 & 33 & 40,2 & 1 & 1,2 \\
\hline
\end{tabular}

Fonte/Source: ESF Bayeux - PB/ Abril-Maio 2007.

Tabela 8 - Valor da Mediana para o componente "Acesso" por Distrito de Saúde no município de Bayeux, PB.

Table 8 - Median Value of the "Access" component per Health District. Bayeux, PB

\begin{tabular}{lccccc}
\hline Indicadores & I & II & III & IV & V \\
\hline O doente realiza a coleta de escarro na unidade? & 1 & 1 & 1 & 2 & 2,5 \\
Se o doente necessitar de atendimento no horário de almoço, ele é atendido? & 4,5 & 1 & 2 & 2 & 1,5 \\
Os doentes de TB podem conseguir uma consulta nesta unidade? & 6 & 6 & 6 & 6 & 6 \\
Os doentes de TB podem obter uma consulta médica no prazo de 24 horas? & 6 & 6 & 6 & 6 & 6 \\
Durante os 6 últimos meses, com que frequência a unidade teve suprimento e & 6 & 6 & 4 & 6 & 6 \\
oferta adequada de medicamentos para o tratamento da TB? & & & & & 1 \\
A unidade oferece vale-transporte para o doente vir até aqui para fazer o TS? & 1 & 1 & 1 & 1 & 1 \\
Com que frequência você vai ao domicílio do doente? & 3 & 6 & 5 & 3 & 4 \\
\hline
\end{tabular}

A Tabela 8 apresenta os indicadores de acesso estudados a partir do valor mediano encontrado por Distrito de Saúde. O acesso ao diagnóstico e tratamento foi classificado segundo os valores obtidos dos indicadores. Atribuiu-se aos valores próximos de 1 e 2 a classificação de acesso não-satisfatório, próximo de 3 e 4 regular, e próximo de 5 e 6 satisfatório.

Em relação à realização de coleta de escarro na USF, o valor da mediana apresentou escores iguais ou inferiores a 2,5 em todos os Distritos de Saúde representando que o acesso à realização de exame para diagnóstico e controle de TB é pouco incorporado pelas ESF.

Verificou-se que, em todos os Distritos, o acesso dos pacientes de TB à consulta médica foi satisfatório (valores medianos igual a 6) e que a mesma pode ser conseguida em um prazo de 24 horas. Entretanto, verificaram-se restrições de atendimento no horário de almoço. O Distrito I apresentou acesso regular, valor mediano igual a 4,5 , e os demais Distritos valores medianos iguais ou inferiores a 2, mostrando acesso não satisfatório para este indicador

No que tange ao suprimento e à oferta adequada de medicamentos, evidenciou-se acesso satisfatório (valor da mediana igual a 6) para a maioria dos Distritos de Saúde. Somente o Distrito III apresentou valor da mediana inferior (4), representando acesso regular aos medicamentos para tratamento de TB.

Quanto à disponibilização de auxíliotransporte ao paciente para realização do tratamento supervisionado na Unidade, 
verificou-se acesso não satisfatório em todos os Distritos investigados (valor de mediana igual a 1 )

Em relação à frequência com que as equipes comparecem ao domicílio do doente, observou-se que esta não é uma prática homogênea entre os Distritos de Saúde. Os Distritos II e III destacaram-se apresentando acesso satisfatório, valores medianos iguais a 6 e 5, respectivamente, e os Distritos I , IV e V acesso regular, com valores medianos entre 3 e 4 .

\section{Discussão}

A ampliação da realização de baciloscopia para a rede de serviços de saúde, visa prioritariamente detectar os doentes de tuberculose e iniciar precocemente o tratamento dos casos. Uma vez que este procedimento se caracteriza como uma ação prevista para ser executada pelas ESF, seria relevante compreender quais fatores estariam dificultando a inserção desta prática no cotidiano de trabalho das equipes de saúde da família no município estudado. Sugere-se que este pode ser indicativo de deficiências organizacionais (previsão de insumos, capacitação insuficiente dos profissionais para realização do procedimento) que repercutem no desempenho das ESF.

Nesta perspectiva, merecem destaque dois pontos fundamentais e estruturantes para o bom desempenho das equipes. $\mathrm{O}$ primeiro se relaciona à organização da rede de serviços na "meso-gestão". Refere-se às formas de organizar os sistemas de saúde, de modo a atender as demandas por serviços e as necessidades de saúde. O segundo se relaciona à capacidade de interação do doente na "micro-gestão". Estes elementos são descritos como "componentes estruturais" que podem ser utilizados para criar ou redesenhar um sistema de saúde capaz de gerir com maior eficácia os problemas de saúde de longo prazo ${ }^{11}$.

Do ponto de vista da meso-gestão, no que tange ao diagnóstico da tuberculose, são necessários mecanismos que garantam o fluxo laboratorial do material coletado (armazenamento, transporte, sistema de informação). Quanto ao componente da "micro-gestão", implica a ampliação da capacidade técnica dos profissionais e o consequente empoderamento de suas funções e competências frente aos casos de tuberculose.

Destaca-se que a descentralização das ações de controle da tuberculose para o âmbito das ESF requer envolvimento dos atores-chave nos níveis central e periférico do sistema de saúde, uma vez que não é possível viabilizar a descentralização das ações sem uma disposição em delegar responsabilidades e sem uma efetiva participação dos atores-chave nos diferentes níveis ${ }^{12}$. As ações precisam ser operacionalizadas e desenvolvidas de igual modo entre os diferentes atores, de acordo com as responsabilidades inerentes à sua função.

Portanto, ressalta-se que a insuficiente oferta do procedimento de coleta de escarro pelas ESF exige deslocamento dos casos suspeitos - ou em tratamento - de tuberculose para outros serviços. Ao transferir para os pacientes a responsabilidade de proceder à coleta e encarregá-los de levar o material ao local estabelecido, coloca-se em risco a ação planejada, comprometendo a integralidade da atenção. Logo, evidencia-se que, apesar da agilidade em se conseguir uma consulta médica, o diagnóstico e controle dos casos de TB podem estar sendo prejudicados frente às dificuldades de acesso ao exame.

Acresce-se que o horário de funcionamento dos Distritos pode dificultar o atendimento de trabalhadores. Os horários rígidos e limitados e as formas quase sempre presenciais de marcação de consultas funcionam como constrangimento ao efetivo acesso à atenção básica, especialmente nas metrópoles, em que parcelas importantes da população passam o dia longe de seu local de moradia ${ }^{5}$. Portanto, os serviços de saúde devem adotar medidas que facilitem o acesso do usuário e favoreçam o atendimento com presteza, o que certamente melhoraria e facilitaria a adesão do paciente ao tratamento ${ }^{13}$. 
Por conseguinte, cabe ao gestor e às equipes locais criar dispositivos operacionais que atendam às necessidades da população. Os serviços devem ser prestados oportunamente e em caráter contínuo, bem como estar disponíveis a qualquer momento, atender à demanda real e incluir um sistema de referência que assegure fácil acesso ao nível de assistência que se requer (acessibilidade funcional) ${ }^{14}$. É preciso reduzir os conflitos existentes entre os padrões técnicos e administrativos dos serviços e os hábitos, padrões culturais e costumes das comunidades em que sejam prestados (acessibilidade cultural).

A dinâmica que se estabelece entre clientela e serviço, ou entre a demanda e a oferta, é realimentada pelo grau de resolutividade dos serviços de saúde e pelo grau de satisfação dos usuários, enfatizando a importância de se analisar o motivo e a frequência da utilização dos serviços, muitas vezes denominados "inacessíveis" ou "indisponíveis" para a população que os procura. Ressalta-se que as ações de saúde devem ser compostas e operadas de modo articulado e integrado, de tal forma que permita ampla cobertura e acesso da população, com a maior eficiência econômica e social possível ${ }^{15}$.

Com relação ao acesso aos medicamentos para tratamento da TB, evidenciou-se que o mesmo é satisfatório na maioria dos Distritos, com exceção do Distrito III. Em estudo realizado no ano de 2006, que analisou a descentralização das atividades técnicas e gerenciais do PCT para os municípios prioritários da Paraíba (dentre estes o município de Bayeux), foi evidenciado como potencialidade $\mathrm{o}$ abastecimento regular de medicamentos para o tratamento da $\mathrm{TB}^{4}$. A este respeito, questiona-se se houve mudanças no abastecimento de medicamentos, ou se a fragilidade observada no Distrito III é ocasionada por deficiências locais no gerenciamento desta ação. Considerando que, no Brasil, os esquemas terapêuticos são padronizados, custeados pelo governo e distribuídos aos municípios, seria esperado que todas as ESF dispusessem prontamente de tal recurso. Presume-se que o acesso gratuito a medicamentos para TB seja um dos fatores que corroboram para adesão dos pacientes ao tratamento. Nesta direção, chama a atenção a capacidade organizacional do sistema de serviços de saúde (planejamento, previsão de medicamentos, distribuição, controle de estoque) em atender às demandas geradas no cotidiano de trabalho das ESF.

Embora esteja prescrito como princípio básico da estratégia saúde da família que as ESF atuem em território, com área de abrangência adstrita, deve-se considerar que os municípios são heterogêneos, com áreas de difícil acesso e realidades geográficas diferenciadas. Portanto, a acessibilidade geográfica é medida pela distância entre a população e os recursos, tempo de deslocamento e custo do transporte ${ }^{16}$. Quando se dispõe de serviços descentralizados, e mais próximos à população, o deslocamento é facilitado.

Ainda que se reconheça que a disponibilização de benefícios (vale-transporte) se caracteriza como um instrumento facilitador para o comparecimento diário do paciente de TB ao tratamento supervisionado, a qualidade da atenção dispensada e o grau de satisfação do usuário devem ser considerados. Alguns autores constataram que a distância entre a residência do doente e a unidade de saúde pode ser um fator dificultante para a adesão terapêutica, embora não seja a causa mais relevante ${ }^{17-21}$. Nesta direção apontam que o acolhimento, como acesso, passa a ser sinônimo de uso, pois não abrange qualquer uso, e sim aquele que ocorre no momento adequado ao entendimento do problema de saúde do usuário, empregando recursos corretos e executando de forma correta ${ }^{22}$.

Por outro lado, a adoção regular da visita domiciliar tornaria dispensável o emprego do auxílio-transporte. Contudo, esta prática não é adotada regularmente pelos profissionais das ESF pesquisadas. Chama a atenção que a visita domiciliar é uma ferramenta de trabalho das ESF e condição essencial para o acompanhamento dos casos de TB em regime supervisionado. A aproximação com 
a realidade dos pacientes favorece a convivência dos profissionais com a dinâmica do processo de adoecimento e cura no meio popular, bem como o confronto com a complexidade dos problemas de saúde dessas populações, levando muitos profissionais a buscarem a reorientação de suas práticas com a finalidade de enfrentar, de forma mais global, os problemas de saúde identificados, rompendo, assim, com a verticalidade da relação profissional-usuário ${ }^{23}$.

\section{Considerações finais}

A aplicação desta metodologia de avaliação foi capaz de identificar as fragilidades e potencialidades de acesso ao diagnóstico e tratamento da Tuberculose em Unidades de Saúde da Família no município de Bayeux. Verifica-se que ainda existem desafios a serem enfrentados que fortaleçam a capacidade organizacional das ESF e resultem em maior resolutividade da atenção. Constatase que:

- a descentralização do tratamento e acompanhamento dos casos de tuberculose vem se estabelecendo na prática das ESF;

- as ESF contam com suprimento adequado de medicamentos para o tratamento da TB;

- o procedimento prioritário para a realização do diagnóstico (coleta de escarro) não é realizado pelas ESF;

- as equipes não contam com benefícios (vale-transporte) como incentivo para promover maior adesão dos pacientes ao tratamento;

- nem todos os profissionais das ESF adotam a visita domiciliar como rotina de trabalho;

- as ESF, em sua maioria, não oferecem atendimento ao paciente de TB no horário de almoço.

Acrescenta-se que a análise dos indicadores evidenciou posicionamentos diferenciados entre os entrevistados dos mesmos Distritos de Saúde, demonstrando que as ESF operam com capacidades organizacio- nais distintas. Aponta para necessidade de ajustes, seja no planejamento e/ou organização das ações, cujo enfoque privilegie o cumprimento adequado das prescrições oficiais determinadas pelo Programa Nacional de Controle da Tuberculose e em conformidade com os objetivos da Atenção Primária à Saúde.

Concebe-se, entretanto, que os resultados dessa avaliação não se traduzem automaticamente em uma decisão, mas espera-se que as informações produzidas contribuam para a validação do julgamento e influenciem positivamente a decisão.

Merecem destaque os seguintes apontamentos:

- Sugere-se que, em futuras pesquisas, seja incluído um maior número de informantes-chave (profissionais, usuários, gestores) para ampliar a capacidade de análise, bem como a aplicação simultânea desta metodologia em outros municípios na perspectiva de comparar os resultados e identificar as potencialidades e fragilidades experienciadas em diferentes cenários, possibilitando correlações estatísticas.

- Recomenda-se a realização de estudos que possibilitem a avaliação de outros componentes da APS (vínculo, coordenação, enfoque familiar, orientação para a comunidade) que consistem em importantes elementos para medir o desempenho dos serviços de saúde.

- Recomenda-se a adoção de mecanismos de gestão que viabilizem a uniformização de recursos estruturais, amplie a capacidade resolutiva das ESF, promova a eficiência na prestação de serviços e assegure o acesso da população.

Os achados permitem concluir que a superação das deficiências requer convencimento e esforços de diferentes atores do sistema de serviços de saúde. Ao gestor compete o planejamento e a distribuição eficiente dos recursos. Todavia, é sabido que a disponibilidade de um serviço, ou sua simples existência, não indica grupo 
populacional coberto nem a extensão da cobertura. Assim, chama a atenção que a eficiência da utilização dos recursos vai exigir profissionais compromissados, envolvidos e conscientes de sua responsabilidade.

Neste sentido é importante acrescentar que a metodologia de avaliação deve ser empregada não apenas para monitorar as características organizacionais dos serviços de saúde, mas também as possíveis mudanças na atuação e, ao longo do tempo, avançar na avaliação do impacto dessas mudanças na saúde da população.

\section{Referências}

1. Macinko J, Almeida C, Oliveira E. Avaliação das características organizacionais dos serviços de atenção básica em Petrópolis: teste de uma metodologia. Saúde em Debate 2003; 27(65): 243-56.

2. Ruffino Netto A. Impacto da reforma do setor saúde sobre os serviços de tuberculose no Brasil. Bol Pneumol Sanit; 1999.

3. Brasil. Ministério da Saúde. Tuberculose: guia de Vigilância Epidemiológica. Brasília: Fundação Nacional de Saúde; 2002.

4. Sá LD, Figueiredo TMRM, Lima DS, Andrade MN, Queiroga RP, Cardoso MAA. et al. A experiência da implantação da estratégia DOTS em seis municípios paraibanos. In: Ruffino Uffino Netto A, Villa TCS. Tuberculose - Implantação do DOTS em Algumas Regiões do Brasil: Histórico e Peculiaridades Regionais. Ribeirão Preto: FMRP; 2006. pp. 141-66.

5. Macinko J, Viacava F, Almeida C, Caetano R, Fausto M, Martins M. et al. Uma Metodologia de Avaliação do Desempenho do Sistema de Saúde Brasileiro. Cien Saúde Colet 2004; 8(3).

6. Felisberto E. Da teoria à formulação de uma Política Institucional de Avaliação em Saúde: reabrindo o debate. Cien Saúde Colet 2006;11(3): 553-63.

7. Pisco LA. A avaliação como instrumento de mudança. Cien Saúde Colet 2006; 11(3): 564-76.

8. IBGE. Fundação Instituto Brasileiro de Geografia e Estatística. Mapas Base dos Municípios do Estado da Paraíba. Escalas variadas, 2004. Disponível em http:// dtr202.saude.gov/br/ caadab/indicadores/paraíba/ BAYEUX.pdf. [Acessado em 28 de outubro de 2007].

9. Almeida C, Macinko J. Validação de uma metodologia de avaliação rápida das características organizacionais e de desempenho dos serviços de atenção básica do Sistema Único de Saúde (SUS) em nível local. Brasília: OPAS/ OMS; 2006.
10. Villa TCS, Ruffino Netto A. Instrumento adaptado pela avaliação das dimensões organizacionais e de desempenho na atenção básica para o controle da tuberculose. EERP/USP E FMRP/USP-REDE-TB; 2007.

11. Organização Pan-Americana de Saúde/Organização Mundial de Saúde. Oficina Sanitária Panamericana, Oficina Regional de la Organización de la Salud, Washington; 2002; 5(1).

12. Ruffino Netto A, Villa TCS. Tuberculose - Implantação do DOTS em algumas regiões do Brasil: histórico $e$ peculiaridades regionais. Ribeirão Preto: FMRP; 2006.

13. Morrone N, Solha MSS, Cruvinel MC, Freire JAS, Barbosa ZLM. Tuberculose: tratamento supervisionado "vs" tratamento autoadministrado. Jornal de Pneumol 1999; 25(4): 198-206.

14. Almeida ES, Zioni F, Chioro A. Estado, Políticas Públicas e Sistema de Saúde: antecedentes, Reforma Sanitária e o SUS; 1998. (Texto preliminar, mimeo).

15. Goldbaum M, Gianini RJ, Novaes HMD, César CLG. Utilização de serviços de saúde em áreas cobertas pelo programa saúde da família no Município de São Paulo. Rev Saúde Pública 2005; 39(1): 90-9.

16. Starfield B. Atenção primária: equilíbrio entre necessidades de saúde, serviços e tecnologia. Brasília: UNESCO, Ministério da Saúde; 2002.

17. Gonçalves H, Costa JSD, Menezes AMB, Knauth D, Leal OF. Adesão à terapêutica da tuberculose em Pelotas, Rio Grande do Sul: na perspectiva do paciente. Cad Saúde Publica 1999; 15(4): 777-87.

18. Oliveira HB. Estudo do tratamento da tuberculose na Rede Pública no município de Campinas. 1991. [dissertação de mestrado], Faculdade de Medicina da Universidade Estadual de Campinas: Campinas: 1991.

19. Natal S, Revisão bibliográfica - Tratamento da tuberculose: causas de não aderência. Bol de Pneumol Sanit 1997; 5: 51-71. 
20. Natal S, Elias MV. Projeto de Análise de informação para a tuberculose. Bol de Pneumol Sanit 2000; 8: 15-22.

21. Reigota SN. Avaliação do controle da tuberculose pulmonar no município de Bauru-SP - Implantação do tratamento supervisionado: 1999/2000. [dissertação de mestrado], Universidade Estadual de São Paulo: São Paulo: 2001.

22. Travassos C, Martins M. Uma revisão sobre os conceitos de acesso e utilização de serviços de saúde. Cad Saúde Pública 2004; 20: 190-8.
23. Alves VS. Um modelo de educação em saúde para o Programa de Saúde da Família: pela integralidade da atenção e reorientação do modelo assistencial. Interface 2005; 9(16):

Recebido em: 1/12/07

Versão final reapresentada em: 16/10/08

Aprovado em: 16/04/09 